\title{
Timing matters: the underappreciated role of temperature ramp rate for shape control and reproducibility of quantum dot synthesis $\uparrow$
}

\author{
William J. Baumgardner, ${ }^{* a}$ Zewei Quan, ${ }^{b}$ Jiye Fang ${ }^{b}$ and Tobias Hanrath ${ }^{c}$
}

Received 30th April 2012, Accepted 14th May 2012

DOI: $10.1039 / \mathrm{c} 2 \mathrm{nr} 31054 \mathrm{f}$

Understanding the coupled kinetic and thermodynamics factors governing colloidal nanocrystals nucleation and growth are critical factors in the predictable and reproducible synthesis of advanced nanomaterials. We show that the temporal temperature profile is decisive in tuning the particle shape from pseudo-spherical to monodisperse cubes. The shape of the nanocrystals was characterized by transmission electron microscopy and X-ray diffraction. We introduce a mechanism for the shape controlled synthesis in the context of temperature-dependent nucleation and growth and provide experimental evidence to support it.

Lead chalcogenides nanocrystals (NCs) have been the subject of intense research efforts because they provide an advantageous model system for fundamental quantum confinement studies as well as building blocks for NC-based devices. An impressively broad range of $\mathrm{NC}$ shapes having been reported in the literature, including spheres, cubes, octahedra, rods, and stars. ${ }^{1-3}$ The various NC shapes can be achieved through relatively minor modifications in the synthesis conditions, e.g. changing the composition of the surfactant mixture. ${ }^{1}$ Several studies have recently been reported detailing the effects of acetate content ${ }^{4}$ trioctylphosphine impurities, ${ }^{5}$ oxidation of solvent double bonds, ${ }^{6}$ and phosphine chemistry of selenium precursors $^{7}$ on particle yield, size, and shape. Unfortunately, the sensitive response to synthesis conditions in these and other materials has often led to a lack of reproducibility from run-to-run and from lab-to-lab. There is a growing recognition in the field that a more detailed approach to the characterization and reporting of synthetic procedures is required to fully standardize the production of nanomaterials. ${ }^{8}$ To address this issue, we investigated the time-temperature profile of the hot-injection synthesis. Our results provide new insights into the underlying kinetic and thermodynamic considerations governing shaped controlled NC synthesis. We demonstrate how tuning the time-temperature profile affords robust control over

${ }^{a}$ Baker Laboratory, Cornell University, Ithaca, New York, USA. E-mail: wjb87@cornell.edu

${ }^{b}$ Chemistry Department, State University of New York at Binghamton, Binghamton, New York, USA

'School of Chemical and Biomolecular Engineering, Cornell University, Ithaca, New York, USA. E-mail: th358@cornell.edu

$\dagger$ Electronic supplementary information (ESI) available: Experimental details, temperature dip experiments, and water content experiments. See DOI: $10.1039 / \mathrm{c} 2 \mathrm{nr} 31054 \mathrm{f}$ the shape of PbSe NCs from pseudo-spherical to cubic without alteration of any other synthetic parameters. Importantly, this approach provides shape control without altering the NC surface chemistry, which is an important prerequisite for the study of the NC shape dependent electronic structure.

The rock salt crystal structure of lead chalcogenide NCs facilitates direct correlation of crystal shape to the growth of specific facets. ${ }^{2,3}$ In general, the shape of a NC depends on the relative rate at which specific facets grow during synthesis. The growth rate can be related to the surface energy of a particular facet via the Curie Gibbs Wulff theorem. ${ }^{9}$ Although this theorem applies to systems in thermodynamic equilibrium, it provides a helpful basis for explaining shape controlled NC growth. ${ }^{10}$ The specific challenge for the growth of cubic lead salt NCs is therefore to achieve fast growth of high surface energy (111) facets to yield a crystal shape defined by low energy (100) facets. While theoretical studies of capped and uncapped surfaces have been reported, many questions remain about the physical and chemical nature of NC surfaces and the bound ligand. ${ }^{11,12}$ Interest in shape controlled lead salt $\mathrm{NC}$ synthesis derives in large part from the influence of shape on the electronic structure of individual $\mathrm{NCs}^{13}$ and the inter-NC coupling in condensed films. ${ }^{14}$

We synthesized cubic PbSe NCs according to a simplified method loosely based on that reported by Lu et $a l^{2}$ Special care was taken to remove trace water from solvents and precursor solutions (see ESI $\dagger$ ). A lead oleate stock solution was produced by dissolving $6 \mathrm{mmol}$ of $\mathrm{PbO}$ in $48 \mathrm{~mL}$ of diphenyl ether (DPE) and $12 \mathrm{~mL}$ of oleic acid at elevated temperatures, which was then cooled and stored under nitrogen for later use. Separately, $8 \mathrm{~mL}$ of DPE and $3 \mathrm{~mL}$ of $1 \mathrm{M}$ trioctylphosphine : selenide (TOP : Se) were heated to $200{ }^{\circ} \mathrm{C}$ under nitrogen for one hour. $10 \mathrm{~mL}$ of lead oleate stock solution and $2 \mathrm{~mL}$ of room temperature DPE were simultaneously rapidly injected into the reaction mixture, which caused the reaction temperature to dip below $140{ }^{\circ} \mathrm{C}$. The solution was heated back up to $200^{\circ} \mathrm{C}$ at varying rates and NCs were allowed to grow for 6 minutes before quenching. The NCs were cleaned using hexane and ethanol as the solvent and anti-solvent respectively.

We performed a series of reactions under identical starting conditions while adjusting the temperature ramp rate following the precursor injection. This approach allowed us to isolate the effects of the temperature curve on particle shape. Fig. 1 shows the complete time-temperature profiles for these reactions, along with transmission electron microscopy (TEM) images of the particles. Importantly, the TEM data clearly illustrate that the controlled temperature profile 

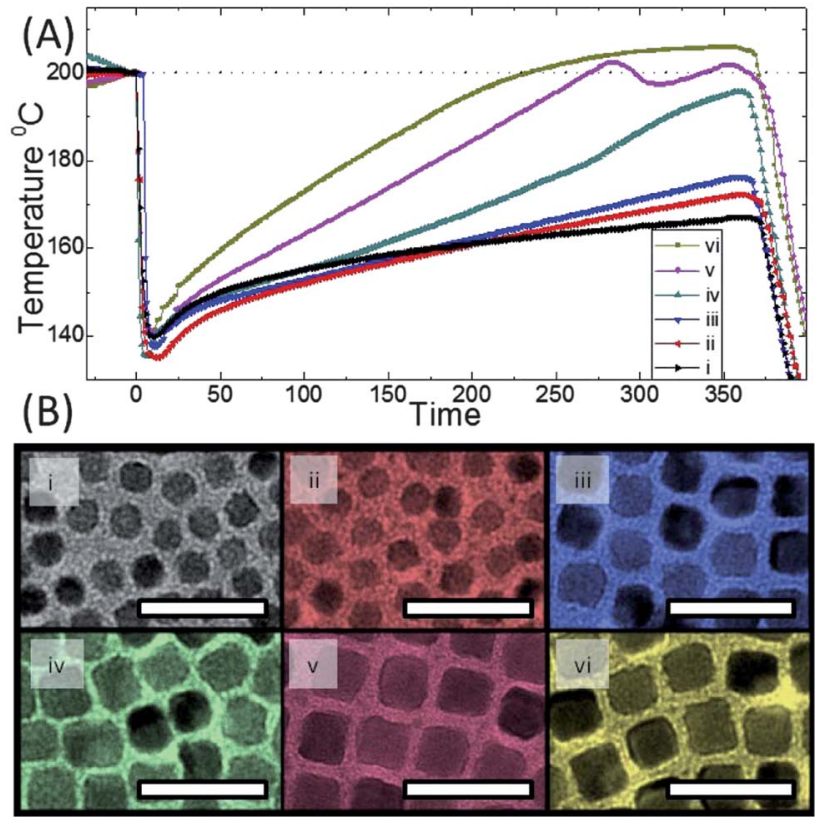

Fig. 1 (A) Detailed reaction time-temperature profile of six different reactions with identical starting conditions, labeled (i)-(vi). (B) TEM images of reactions (i)-(vi) (scale bar: $30 \mathrm{~nm}$ ).

yields control over NC shape ranging from pseudo-spherical to cubic with increasing temperature ramp rates. We note that because in typical literature reports detailed aspects of the time-temperature profile are generally not included, in many cases each of these reaction protocols would be described nearly identically. Particles synthesized with the highest ramp rates yielded $11.1 \mathrm{~nm}$ monodisperse cubes with an average aspect ratio of 1.08 and a size distribution below $9 \%$.

Accurate and representative analysis of the $\mathrm{NC}$ shape requires special experimental consideration. TEM provides helpful first insights, but shape analysis is complicated by the fact that the micrograph is a two-dimensional projection of a three-dimensional structure and moreover influenced by sampling bias since only a very small fraction of the sample, usually the very best images, are included in the analysis. The form factor derived from X-ray scattering analysis can, in principle, be used to assess the NC shape. However, variations in NC size distribution obscure the direct shape analysis from their scattering pattern. To gain reliable information about particle shape, we exploited the fact that NC shape influences their self-assembly in thin films. Specifically, we exploited the ratio of the (200) : (111) reflections as a convenient metric of the orientational ordering of $\mathrm{NC}$ in the film and hence the cubic character of the $\mathrm{NC}$ shape. NC films with approximately $60 \mathrm{~nm}$ thickness were prepared by drop casting a dilute solution ( $1 \mathrm{mg} \mathrm{mL}^{-1}$ in hexane) of NCs onto precleaned silicon substrates and allowing rapid evaporation $(<1$ minute) of the solvent.

Fig. 2 illustrates the direct correlation between the between the ratio of (200) : (100), i.e. the cubic NC shape and the temperature ramp rate. Similar diffractograms have been observed previously, and indicate a specific orientation of the particle to the substrate. ${ }^{15}$ Spherical NCs do not exhibit preferred crystallographic orientation relative to the substrate and yield powder-like X-ray diffraction patterns. In the case of cubes, large (100) facets preferentially lie flat
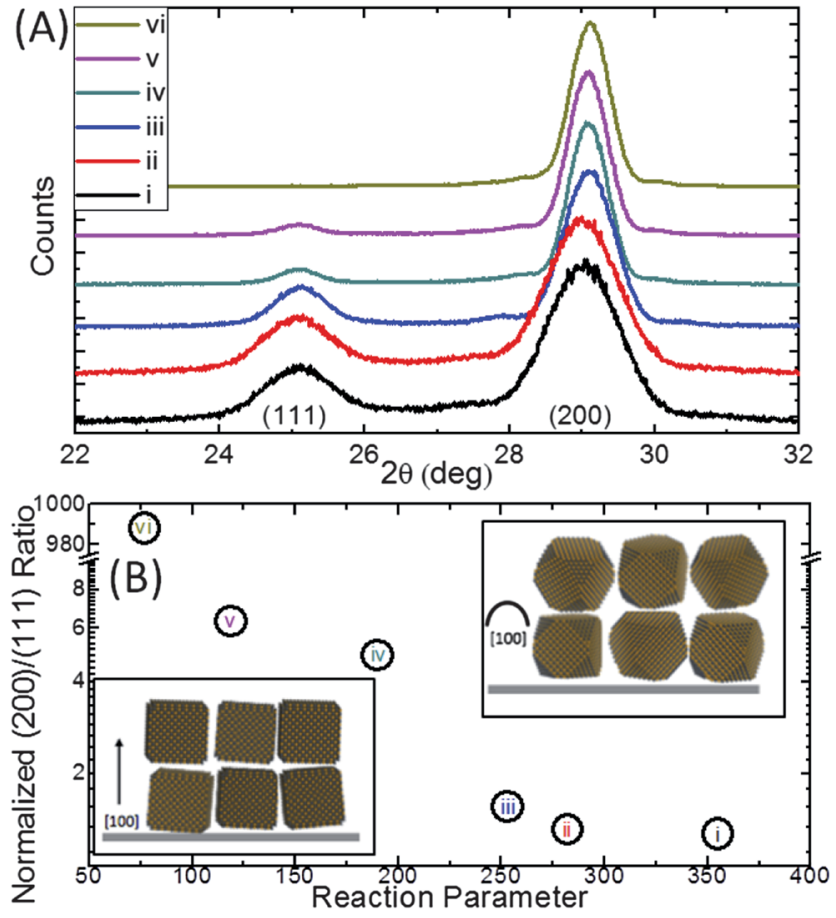

Fig. 2 (A) X-ray diffraction of samples (i)-(vi) from Fig. 1 with PbSe rock-salt (111) and (200) indexed. (B) Integrated ratio of the (200) : (111) reflections normalized to the powder value plotted against the reaction parameter, defined as the time in seconds after initial injection that the reaction mixture reached $167{ }^{\circ} \mathrm{C}$. Schematics with the random and preferential orientation of nanocrystals corresponding to the X-ray response are included as well.

on the substrate which leads to a preferred crystallographic orientation. As a result, for highly cubic particles, X-ray reflections from (100) planes will be more pronounced, which is the trend we report here. Samples (i)-(iii) exhibit a powder-like diffraction pattern without preferential NC orientation. Samples (iv) and (v) exhibit integrated (200): (111) ratios that are 5 and 6 times larger than in the powder indicating preferential orientation of cube faces normal to the substrate. Sample (vi) displays an almost exclusive preference for the (200) reflection, with the (200): (111) ratio at almost 1000. Beyond the TEM analysis, this data shows the cube-like behavior of $\mathrm{PbSe}$ NCs synthesized using a high ramp rate. We will provide a more detailed analysis of the packing structures of nanocubes in an upcoming report.

While our investigations found that a six minute reaction is optimum for the production of cubes that are monodisperse in terms of size and shape, this introduces ambiguity about the source of the shape tunability. Specifically, within the six minute time frame, the reactions with slower ramp rates, such as (i)-(iii), do not reach the same final growth temperature as the (iv)-(vi). This raises an important question: is it the ramp rate that influence the particle shape or only the final growth temperature? To address this issue we considered two sets on reactions. In the first set (Fig. S1 $\dagger$ ), we extended the growth time to 30 minutes and changed the final reaction temperature to $180{ }^{\circ} \mathrm{C}$. Despite all reactions reaching the same temperature by the end of their growth period (in most cases for several minutes), the products still represented a continuum from pseudo-spherical to cubic as temperature ramp rate increased. 


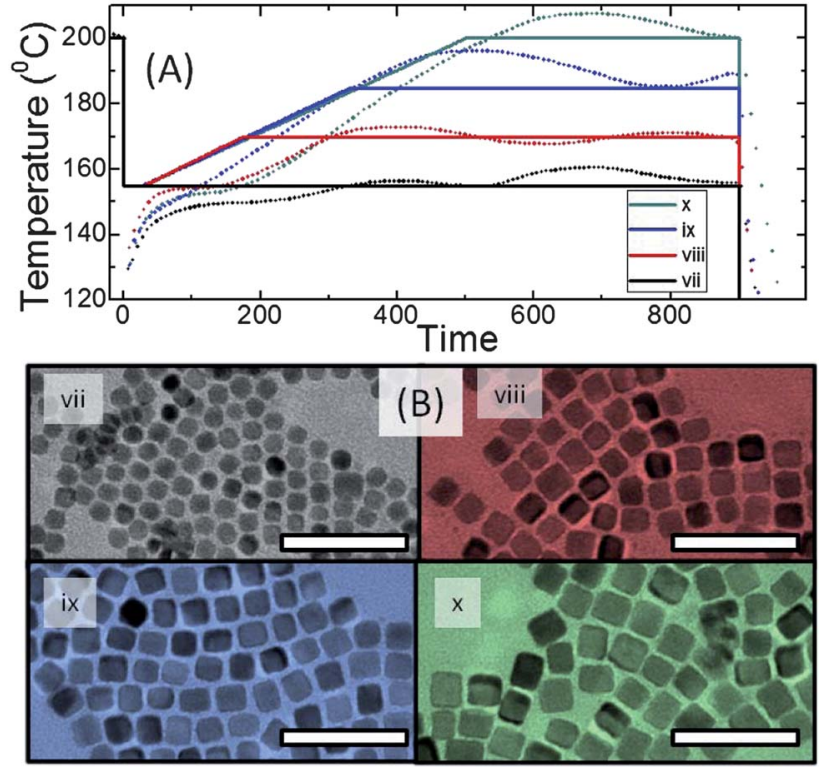

Fig. 3 (A) Time temperature profiles of 4 different reactions with identical starting conditions labeled (vii)-(x). Solid lines indicate the set points on the temperature controller while dotted lines are the measured values. (B) TEM images of reactions (vii)-(x) (scale bar: $50 \mathrm{~nm}$ ).

For the reactions in Fig. 3, we used as similar a ramp rate as the dynamic hot injection allows but varied the final growth temperature. These reactions produced cubic particles for a relatively wide range of final growth temperatures $\left(170-200{ }^{\circ} \mathrm{C}\right)$. We note that for reaction (viii), cubes are produced even though the final growth temperature is below that of samples (ii) and (iii), and all of the samples in Fig. S1†. These data show that in order for cubes to be produced, it is not sufficient merely for a high temperature to be reached, but that it must be reached at an early point in the reaction.

A complicating aspect of the hot-injection synthesis is that both synthesis temperature and precursor concentration evolve during the course of the reaction. To decouple the effects of the temperature profile from the depleting precursor concentration we designed experiments with carefully timed sequential precursor injections and time-temperature profiles as shown in Fig. 4. To probe the evolution of the NC shape, we took aliquots at specific reaction times. The shape of NCs after injection but prior to the ramp-up was spherical (sample xi). After ramping up the temperature to $200^{\circ} \mathrm{C}$ (sample xii), the NC shape did not change appreciably over the course of 5 minutes (sample xiii). The nearly spherical NC shape at the high temperature is an indication that the NC shape is not simply a function of synthesis temperature. The ability of the $\mathrm{NC}$ to attain the cubic equilibrium shape is constrained by the lack of precursor and the slow rates of solid shape transformations. Only upon addition of extra precursor (lead : oleate stock solution $(2.5 \mathrm{~mL})$ and TOP : Se $(0.75 \mathrm{~mL}))$ is the spherical shape attained as validated in Fig. 4, sample (xiv). Allowing particles to remain at high temperatures does not result in a shape transformation to cubes, contrary to what has been observed in some other systems, e.g. iron oxide NCs. ${ }^{16}$

We interpret the results of this experiment based on the relative energies of the (100) and (111) surfaces. Theoretical calculations predict that (100) is the most energetically stable surface for rock-salt $\mathrm{PbSe}^{11}$ suggesting that if $\mathrm{PbSe} \mathrm{NCs}$ are allowed to achieve
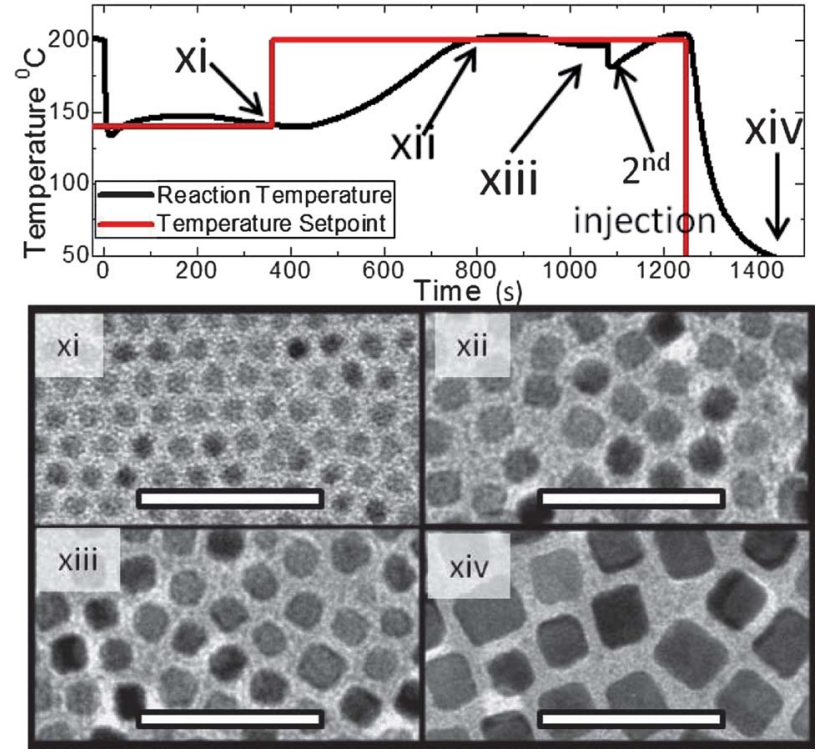

Fig. 4 (Top) the time-temperature profile of a single reaction is reported, along with the time of retrieval of several aliquots of reaction solution. A $2^{\text {nd }}$ injection was performed just after the retrieval of aliquot $\mathrm{C}$. (Below) representative TEM images of aliquots (xi)-(xiv) from the above reaction (scale bar: $50 \mathrm{~nm}$ ).

thermodynamic equilibrium the (100) facets will start to dominate, resulting in a cubic shape. However, if the high temperature necessary to achieve that equilibrium is not attained quickly enough, most of the monomer will have already been depleted in the particle growth, making shape change only possible through the slow route of solid state kinetics. As seen in the previous experiment, little shape change is observed after 6 minutes of growth time at high temperatures, however by injecting a small amount of monomer into the solution, liquid state kinetics again become available, allowing the particles to adopt a thermodynamically favorable shape in a much shorter period of time. The necessity of achieving a high temperature while there is still enough monomer in solution to allow particle growth explains the critical dependence on post-injection ramp rate.

To illustrate the versatility of the NC shape control through programming the time-temperature profile we demonstrate the depth of the temperature dip following the precursor injection as an additional adjustable parameter. We manipulated this temperature dip by either heating the injection solution or by increasing the solvent volume during precursor injection (Fig. S2 $\uparrow$ ). While cubic NCs are produced as long as a high temperature is achieved sufficiently quickly, a smaller temperature dip leads to lower monodispersity and smaller yields, in some cases less than $1 \mathrm{mg}$ of well-dispersed, unaggregated product. This agrees well with previous studies on the importance of a temperature decrease on a single discreet nucleation event ${ }^{17}$ and the fusing of NCs at elevated temperatures. ${ }^{18}$ We also note that while special care has been taken to remove trace water in these reactions, it is possible to produce cubic NCs without these precautions. In fact, the relationship between ramp-rate and particle shape illustrated in our work is remarkably robust to perturbations in the chemical nature of the synthesis environment. The ability to tune $\mathrm{NC}$ shape via time temperature profile held even in the presence of minute amounts of water (Fig. S3†). Moreover, we note that the reaction was rather insensitive to the solvent mixture. Cubes were 
successfully produced in a variety of different solvents, though the products are most monodisperse when made from DPE (Fig. S4 $\dagger$ ).

\section{Conclusions}

We have investigated the effect of the relationship between the timetemperature profile and the growth of $\mathrm{PbSe} \mathrm{NC}$ with programmable shape. We show that precise control of the post-injection ramp rate is critical for shape control and reproducibility of synthesis products. By adjusting the ramp rate independently of other parameters, we were able to tune the shape from pseudo-spherical to monodisperse cubes. We illustrate the necessity of growing cubic NCs in the thermodynamically controlled regime, while still providing enough monomer to allow for particle growth. These insights have important and general implications for the shape control, reproducibility, and reporting of synthesized NCs.

\section{Acknowledgements}

The authors would like to thank Richard Robinson for helpful discussions. This publication was based on work supported by Award No. KUS-C1-018-02, made by King Abdullah University of Science and Technology (KAUST). We also acknowledge the Cornell Center for Materials Science (NSF DMR-0520404).

\section{Notes and references}

1 K.-S. Cho, D. V. Talapin, W. Gaschler and C. B. Murray, J. Am. Chem. Soc., 2005, 127, 7140; W.-k. Koh, A. C. Bartnik, F. W. Wise and C. B. Murray, J. Am. Chem. Soc., 2010, 132, 3909; E. Lifshitz, M. Bashouti, V. Kloper, A. Kigel, M. S. Eisen and S. Berger, Nano Lett., 2003, 3, 857; C. B. Murray, S. Sun, W. Gaschler, H. Doyle, T. A. Betley and C. R. Kagan, IBM J. Res. Dev., 2001, 45, 47.
2 W. Lu, J. Fang, Y. Ding and Z. L. Wang, J. Phys. Chem. B, 2005, 109, 19219.

3 W. W. Yu, J. C. Falkner, B. S. Shih and V. L. Colvin, Chem. Mater., 2004, 16, 3318.

4 A. J. Houtepen, R. Koole, D. Vanmaekelbergh, J. Meeldijk and S. G. Hickey, J. Am. Chem. Soc., 2006, 128, 6792.

5 C. M. Evans, M. E. Evans and T. D. Krauss, J. Am. Chem. Soc., 2010, 391, 395.

6 A. M. Jawaid, D. J. Asunskis and P. T. Snee, ACS Nano, 2011, 5, 6465.

7 W.-k. Koh, Y. Yoon and C. B. Murray, Chem. Mater., 2011, 23, 1825.

8 D. V. Talapin and Y. Yin, J. Mater. Chem., 2011, 21, 11454.

9 G. Wulff, Beitr. Krystallogr. Mineral., 1901, 34, 449.

10 C. R. Bealing, W. J. Baumgardner, J. J. Choi, T. Hanrath and R. G. Hennig, ACS Nano, 2012, 6, 2118.

11 C. Fang, M. A. van Huis, D. L. Vanmaekelbergh and H. W. Zandbergen, ACS Nano, 2009, 4, 211; A. F. Mario Argeri, F. Grassi, L. Marchese and M. Cossi, J. Phys. Chem. C, 2011, 115, 11382; P. Schapotschnikow, M. A. van Huis, H. W. Zandbergen, D. Vanmaekelbergh and T. J. H. Vlugt, Nano Lett., 2010, 664.

12 V. Petkov, I. Moreels, Z. Hens and Y. Ren, Phys. Rev. B: Condens. Matter Mater. Phys., 2010, 81, 241304.

13 J. B. Li and L. W. Wang, Nano Letters, 2003, 3, 1357; A. Bartnik, A. Efros, W. K. Koh, C. Murray and F. Wise, Phys. Rev. B: Condens. Matter Mater. Phys., 2010, 82.

14 F. Dumestre, B. Chaudret, C. Amiens, P. Renaud and P. Fejes, Science, 2004, 303, 821 .

15 J. J. Choi, C. R. Bealing, K. F. Bian, K. J. Hughes, W. Y. Zhang, D. M. Smilgies, R. G. Hennig, J. R. Engstrom and T. Hanrath, J. Am. Chem. Soc., 2011, 133, 3131.

16 G. Salazar-Alvarez, J. Qin, V. Sepelak, I. Bergmann, M. Vasilakaki, K. N. Trohidou, J. D. Ardisson, W. A. A. Macedo, M. Mikhaylova, M. Muhammed, M. D. Baro and J. Nogues, J. Am. Chem. Soc., 2008, 130, 13234.

17 D. V. Talapin, A. L. Rogach, M. Haase and H. Weller, J. Phys. Chem. $B, 2001, \mathbf{1 0 5}, 12278$.

18 M. A. van Huis, L. T. Kunneman, K. Overgaag, Q. Xu, G. Pandraud, H. W. Zandbergen and D. 1. Vanmaekelbergh, Nano Lett., 2008, 8, 3959 . 